\title{
INFALL AND OUTFLOW AROUND THE HH 212 PROTOSTELLAR SYSTEM
}

\author{
ChIN-FeI LeE \\ Harvard-Smithsonian Center for Astrophysics, Submillimeter Array, 645 North A‘ohoku Place, Hilo, HI 96720; cflee@cfa.harvard.edu
}

Paul T. P. Ho, Henrik Beuther, Tyler L. Bourke, and Qizhou Zhang

Harvard-Smithsonian Center for Astrophysics, Submillimeter Array, 60 Garden Street, Cambridge, MA 02138

AND

Naomi Hirano and Hsien Shang

Academia Sinica Institute of Astronomy and Astrophysics, P.O. Box 23-141, Taipei 106, Taiwan Received 2005 September 12; accepted 2005 November 1

\begin{abstract}
$\mathrm{HH} 212$ is a highly collimated jet discovered in $\mathrm{H}_{2}$ powered by a young Class 0 source, IRAS $05413-0104$, in the $\mathrm{L} 1630$ cloud of Orion. We have mapped around it in $1.33 \mathrm{~mm}$ continuum, ${ }^{12} \mathrm{CO}(J=2-1),{ }^{13} \mathrm{CO}(J=2-1), \mathrm{C}^{18} \mathrm{O}$ $(J=2-1)$, and SO $\left(N_{J}=5_{6}-4_{5}\right)$ emission at $\sim 2 " .5$ resolution with the Submillimeter Array. A dust core is seen in the continuum around the source. A flattened envelope is seen in $\mathrm{C}^{18} \mathrm{O}$ around the source in the equator perpendicular to the jet axis, with its inner part seen in ${ }^{13} \mathrm{CO}$. The structure and kinematics of the envelope can be roughly reproduced by a simple edge-on disk model with both infall and rotation. In this model, the density of the disk is assumed to have a power-law index of $p=-1.5$ or -2 , as found in other low-mass envelopes. The envelope seems dynamically infalling toward the source with slow rotation because the kinematics is found to be roughly consistent with a free fall toward the source plus a rotation of a constant specific angular momentum. $\mathrm{A}^{12} \mathrm{CO}$ outflow is seen surrounding the $\mathrm{H}_{2}$ jet, with a narrow waist around the source. Jetlike structures are also seen in ${ }^{12} \mathrm{CO}$ near the source aligned with the $\mathrm{H}_{2}$ jet at high velocities. The morphological relationship between the $\mathrm{H}_{2}$ jet and the ${ }^{12} \mathrm{CO}$ outflow, and the kinematics of the ${ }^{12} \mathrm{CO}$ outflow along the jet axis, are both consistent with those seen in a jet-driven bow shock model. SO emission is seen around the source and the $\mathrm{H}_{2}$ knotty shocks in the south, tracing shocked emission around them.
\end{abstract}

Subject headings: ISM: individual (HH 212) — ISM: jets and outflows — stars: formation

Online material: color figures

\section{INTRODUCTION}

Despite recent progress, the detailed physical processes in the early stages of low-mass star formation are still uncertain. Currently, the most detailed and successful model of low-mass star formation is the four-stage model proposed in the late 1980s by Shu et al. (1987). In the first stage, slowly rotating molecular cloud cores form within a molecular cloud as magnetic and turbulent support is lost through ambipolar diffusion. In the second stage, a molecular cloud core becomes dynamically unstable and collapses from the inside out, forming a protostar surrounded by an accretion disk deeply embedded within an infalling envelope of dust and gas. In the third stage, as the protostar continues accreting mass through the accretion disk, a stellar wind breaks out along the rotational axis of the system, creating a bipolar outflow. In the fourth stage, the infall and accretion terminate, leaving a newly formed star surrounded by a circumstellar disk. In this paper, we investigate the protostellar collapse and outflow in the second and third stages, with the Submillimeter Array (SMA) ${ }^{1}$ observations around a young protostar, IRAS 05413-0104, and its remarkable jet $\mathrm{HH} 212$.

IRAS $05413-0104$ is a cold, low-luminosity $\left(\sim 14 L_{\odot}\right)$, low-mass source (Zinnecker et al. 1992), located at a distance of $460 \mathrm{pc}$ in the L1630 cloud of Orion. It is surrounded by a cold $\left(\sim 14 \mathrm{~K}\right.$ ), flattened (with an aspect ratio of $2: 1$ ) rotating $\mathrm{NH}_{3}$

\footnotetext{
${ }^{1}$ The Submillimeter Array is a joint project between the Smithsonian Astrophysical Observatory and the Academia Sinica Institute of Astronomy and Astrophysics and is funded by the Smithsonian Institution and the Academia Sinica.
}

envelope with a diameter of $12,000 \mathrm{AU}$ (Wiseman et al. 2001). It is also detected at $1.1 \mathrm{~mm}$ (Zinnecker et al. 1992) and $1.3 \mathrm{~mm}$ (Chini et al. 1997), with a high ratio of millimeter-wave luminosity to bolometric luminosity. It is thus classified as a Class 0 source, which has much material to be accreted still from the surrounding molecular gas envelope (Andre et al. 2000).

HH 212 is a remarkable jet with a length of $\sim 240^{\prime \prime}(\sim 0.6 \mathrm{pc})$ powered by the IRAS source. It was discovered in the rotationalvibrational $v=1-0 S(1)$ emission line of $\mathrm{H}_{2}$ at $\lambda=2.122 \mu \mathrm{m}$ (Zinnecker et al. 1998). It is highly collimated and symmetric, with matched pairs of shock knots (knotty shocks) and bow shocks on either side of the IRAS source. Strong water masers are seen moving at $\sim 60 \mathrm{~km} \mathrm{~s}^{-1}$ along the jet axis near the IRAS source (Claussen et al. 1998). Shocked $\mathrm{SiO}$ emission is seen along the jet axis (Chapman et al. 2002) and around the source (Gibb et al. 2004). CO outflow is also seen surrounding the $\mathrm{H}_{2}$ jet (Lee et al. 2000). Deep observations in $\mathrm{H}_{2}$ with the ESO Very Large Telescope (VLT) show a pair of diffuse nebulae near the bases of the jet, probably tracing the outflow cavity walls illuminated by the bright knotty shocks around the source (McCaughrean et al. 2002). Based on the relative magnitude of the proper motions and radial velocities of the water masers, the $\mathrm{CO}$ outflow is believed to lie within $5^{\circ}$ of the plane of the sky (Claussen et al. 1998) and is thus an excellent system to investigate the transverse kinematics of outflow.

Here we study in detail the circumstellar envelope around the IRAS source, the molecular outflow surrounding the $\mathrm{H}_{2}$ jet, and the interaction between them, with the SMA observations in $1.33 \mathrm{~mm}$ continuum, ${ }^{12} \mathrm{CO}(J=2-1),{ }^{13} \mathrm{CO}(J=2-1), \mathrm{C}^{18} \mathrm{O}$ 
TABLE 1

Summary of Observations

\begin{tabular}{|c|c|c|c|c|}
\hline Line & $\begin{array}{l}\text { Frequency } \\
\quad(\mathrm{GHz})\end{array}$ & $\begin{array}{c}\text { Beam Size } \\
(\operatorname{arcsec} \times \operatorname{arcsec})\end{array}$ & $\begin{array}{c}\text { Channel Width } \\
\left(\mathrm{km} \mathrm{s}^{-1}\right)\end{array}$ & $\begin{array}{l}\text { Noise Level }^{\mathrm{a}} \sigma \\
\quad\left(\mathrm{Jy} \mathrm{beam}^{-1}\right)\end{array}$ \\
\hline $1.33 \mathrm{~mm}$ continuum....................... & 225.000000 & $2.6 \times 2.3$ & $\ldots$ & $3.5 \mathrm{E}-3$ \\
\hline 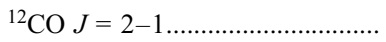 & 230.537980 & $2.8 \times 2.3$ & 0.264 & 0.25 \\
\hline 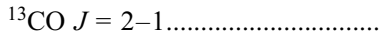 & 220.398676 & $2.8 \times 2.4$ & 0.274 & 0.15 \\
\hline $\mathrm{C}^{18} \mathrm{O} J=2-1$ & 219.560357 & $2.8 \times 2.4$ & 0.274 & 0.14 \\
\hline $\mathrm{SO} J_{K}=6_{5}-5_{4} \ldots \ldots \ldots \ldots \ldots \ldots \ldots \ldots \ldots \ldots \ldots \ldots \ldots \ldots$ & 219.949442 & $2.8 \times 2.4$ & 0.554 & 0.12 \\
\hline
\end{tabular}

${ }^{\text {a }}$ rms noise level per velocity channel.

$(J=2-1)$, and SO $\left(N_{J}=5_{6}-45\right)$ emission at high angular and velocity resolutions. It is currently believed that $1.33 \mathrm{~mm}$ continuum mainly traces dust core, ${ }^{12} \mathrm{CO}$ (particularly in interferometric observations) mainly traces molecular outflow, $\mathrm{C}^{18} \mathrm{O}$ mainly traces envelope, ${ }^{13} \mathrm{CO}$ traces both molecular outflow and envelope, and SO traces mainly shock interactions.

\section{OBSERVATIONS}

Observations in $1.33 \mathrm{~mm}$ continuum, ${ }^{12} \mathrm{CO}(J=2-1),{ }^{13} \mathrm{CO}$ $(J=2-1), \mathrm{C}^{18} \mathrm{O}(J=2-1)$, and SO $\left(N_{J}=5_{6}-4_{5}\right)$ emission toward the IRAS source and the $\mathrm{H}_{2}$ jet were made between 2004 November and 2005 March on top of Mauna Kea with the SMA (Ho et al. 2004) in the compact configuration (see Table 1). Seven antennas were used in the array, giving baselines with projected lengths ranging from 14.1 to $136 \mathrm{~m}$, resulting in a synthesized beam (with natural weighting) with a size of 2". $8 \times 2$ ". 4 at $230 \mathrm{GHz}$. With a shortest projected baseline of $14.1 \mathrm{~m}$, our observations were insensitive to structures more extended than $15^{\prime \prime}(\sim 7000 \mathrm{AU})$ at the $10 \%$ level. The FWHP of the primary beam is $55^{\prime \prime}$, and nine pointings were used to map the whole jet system. The digital correlator was set up with a bandwidth of $104 \mathrm{MHz}$ for each band (or chunk). We used 512 spectral channels for ${ }^{12} \mathrm{CO}, \mathrm{C}^{18} \mathrm{O}$, and ${ }^{13} \mathrm{CO}$ and 256 spectral channels for SO, resulting in a velocity resolution per channel of $\sim 0.27 \mathrm{~km} \mathrm{~s}^{-1}$ for ${ }^{12} \mathrm{CO}, \mathrm{C}^{18} \mathrm{O}$, and ${ }^{13} \mathrm{CO}$ and $0.55 \mathrm{~km} \mathrm{~s}^{-1}$ for SO. The $1.33 \mathrm{~mm}$ (or $225 \mathrm{GHz}$ ) continuum emission was also recorded with a total bandwidth of $\sim 4 \mathrm{GHz}$. In our observation, a phase calibrator (quasar 0423-013) was observed every 20 minutes to calibrate the phases of the source. A planet (Uranus) was observed to calibrate the fluxes. The data were calibrated with the MIR package adapted for the SMA. The calibrated data were processed with the MIRIAD package. The dirty maps that were produced from the calibrated data and corrected for the primary beam attenuation were deconvolved using the Steer clean method. The final channel maps were obtained by convolving the deconvolved maps with a synthesized (Gaussian) beam fitted to the main lobe of the dirty beam. The velocities of the channel maps are local standard of rest.

\section{RESULTS}

In the following, the IRAS source is assumed to have a refined position at $\alpha_{2000.0}=05^{\mathrm{h}} 43^{\mathrm{m}} 51^{\mathrm{s}} .408, \delta_{2000.0}=-01^{\circ} 02^{\prime} 53^{\prime \prime} .13$, which was found at $\lambda=3.5 \mathrm{~cm}$ with the Very Large Array at an angular resolution of 0"3 (Galván-Madrid et al. 2004). The systemic velocity in this region was assumed to be $1.8 \mathrm{~km} \mathrm{~s}^{-1}$ in CO $J=1-0$ observations (Lee et al. 2000) and $1.6 \mathrm{~km} \mathrm{~s}^{-1}$ in $\mathrm{NH}_{3}$ observations (Wiseman et al. 2001). Here it is assumed to be $1.7 \pm 0.1 \mathrm{~km} \mathrm{~s}^{-1}$. Throughout this paper, the observed velocity is the velocity with respect to this systemic velocity. For comparison, our observations are always plotted with the $\mathrm{H}_{2}$ image of the jet recently obtained with the VLT (McCaughrean et al. 2002). The axis of the $\mathrm{H}_{2}$ jet has a position angle of $\sim 23^{\circ}$. The $\mathrm{H}_{2}$ jet is almost in the plane of the sky, with the blueshifted side to the north and the redshifted side to the south of the source.

\section{1. $1.33 \mathrm{~mm}$ Continuum Emission}

Continuum emission is detected around the source at $\lambda=$ $1.33 \mathrm{~mm}$ with a flux of $0.11 \pm 0.03 \mathrm{Jy}$ in between the diffuse nebulae seen in $\mathrm{H}_{2}$ (Fig. 1). This flux is similar to the peak flux
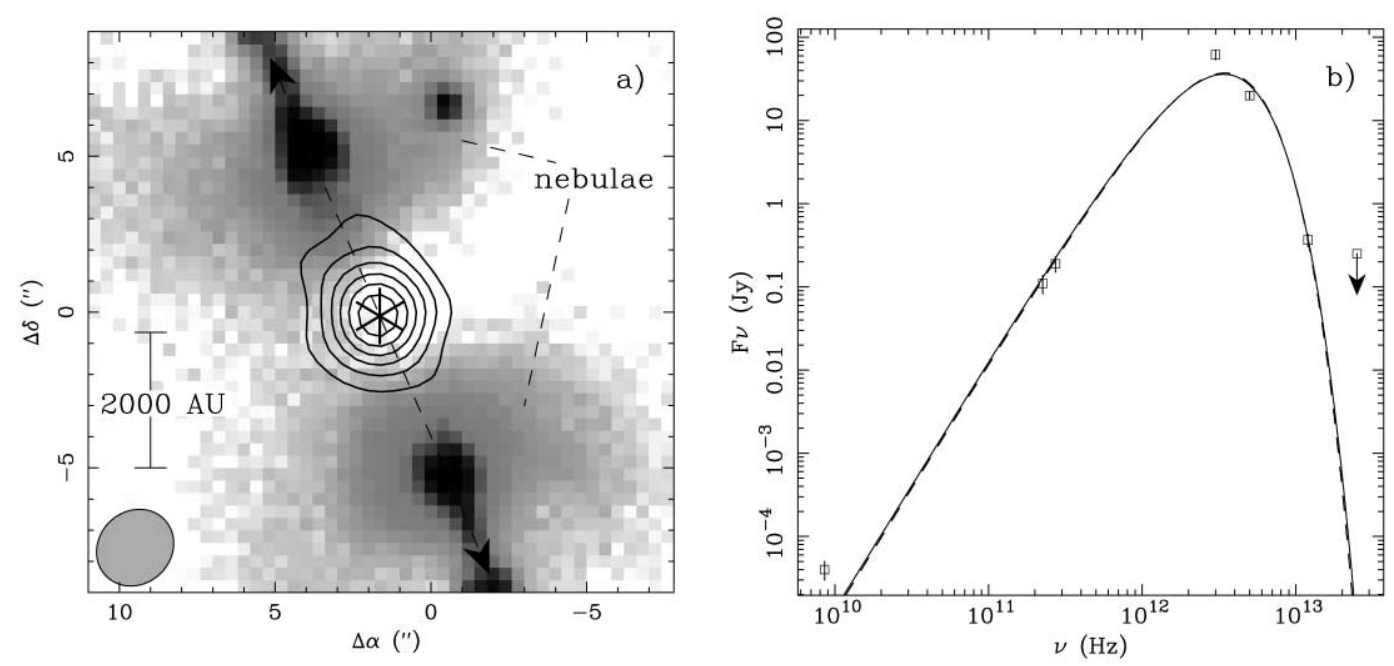

FIG. 1.-(a) $1.33 \mathrm{~mm}$ continuum contours plotted on top of the $\mathrm{H}_{2}$ image adopted from McCaughrean et al. (2002). The contours go from 3 to $18 \sigma$ with a step of $3 \sigma$, where $\sigma=3.5 \mathrm{mJy}$ beam ${ }^{-1}$. The asterisk indicates the IRAS source. Note that the $(0,0)$ position here corresponds to $\alpha_{2000.0}=05^{\mathrm{h}} 43^{\mathrm{m}} 51^{\mathrm{s}} 30, \delta_{2000.0}=$ $-01^{\circ} 02^{\prime} 53^{\prime \prime} 0$. (b) Simple fits to the SED of the continuum source with $\Omega=1.2$ (solid line) and 2.3 (dashed line) $\operatorname{arcsec}^{2}$. 


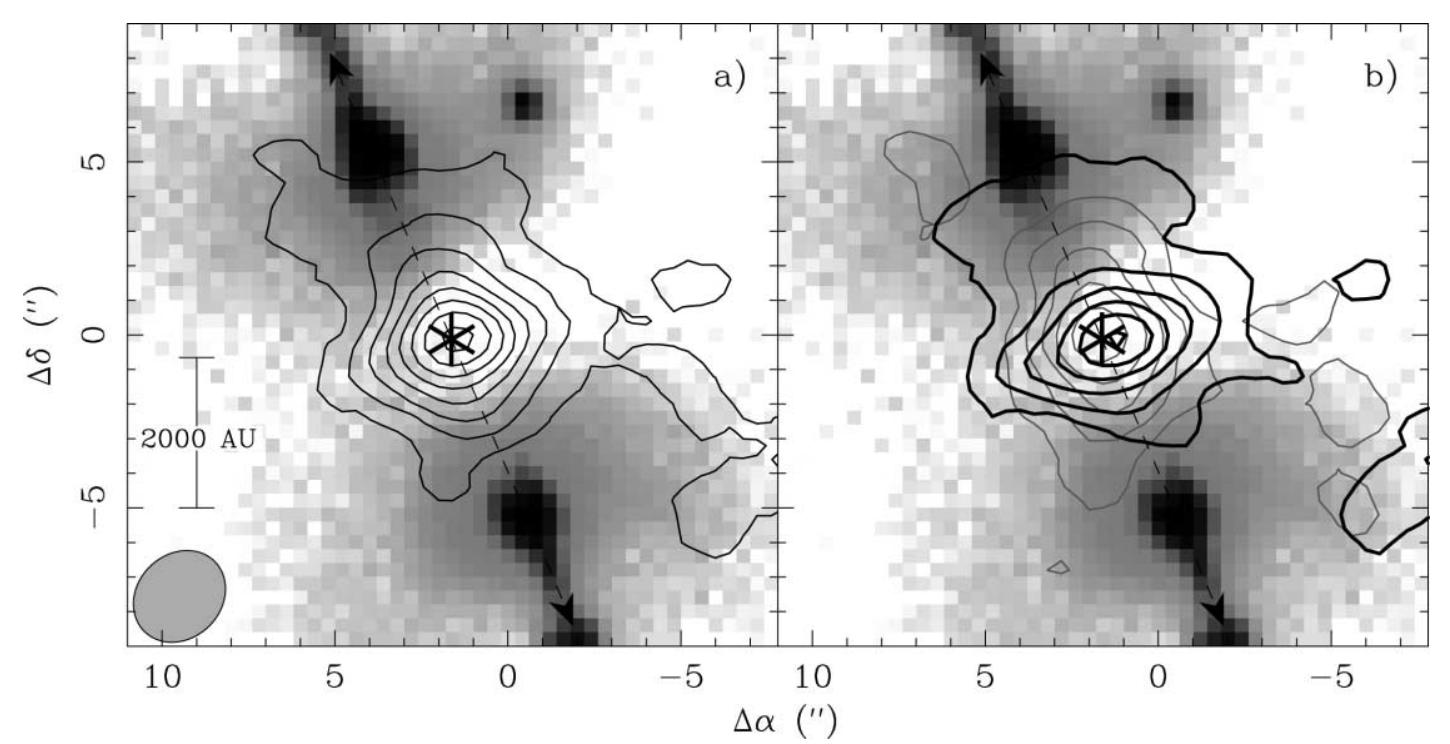

Fig. 2. $-\mathrm{C}^{18} \mathrm{O}$ emission plotted on top of the $\mathrm{H}_{2}$ image, with the asterisk indicating the IRAS source. (a) Total emission integrated over $2.2 \mathrm{~km} \mathrm{~s}{ }^{-1}$ from -1.1 to $1.1 \mathrm{~km} \mathrm{~s}^{-1}$. The contours go from 2 to $23 \sigma$ with a step of $3 \sigma$, where $\sigma=0.11 \mathrm{Jy} \mathrm{beam}^{-1}$. (b) Redshifted (gray contours) and blueshifted (black contours) emission, integrated over $1.1 \mathrm{~km} \mathrm{~s}^{-1}$ on the redshifted and blueshifted sides, respectively. The contours go from 2 to $17 \sigma$ with a step of $3 \sigma$, where $\sigma=0.08 \mathrm{Jy}$ beam ${ }^{-1} \mathrm{~km} \mathrm{~s}^{-1}$. [See the electronic edition of the Journal for a color version of this figure.]

in an $11^{\prime \prime}$ beam around the source found by Chini et al. (1997) with the IRAM 30 telescope. The emission is not resolved and shows a slight elongation along the jet axis. Previously, continuum emission has been detected around the source at $\lambda=1.1 \mathrm{~mm}$ by the James Clerk Maxwell Telescope with a flux of 0.19 Jy (Zinnecker et al. 1992). Continuum emission has also been detected in the infrared by the Infrared Astronomical Satellite (IRAS). The fluxes corrected for extinction are 0.37 , 19.9 , and $61.9 \mathrm{Jy}$ at $\lambda=25,60$, and $100 \mu \mathrm{m}$, respectively. It was not detected at $\lambda=12 \mu \mathrm{m}$. The spectral energy distribution (SED; see Fig. $1 b$ ) is consistent with a Class 0 source. It clearly suggests that the continuum emission at $\lambda=1.33 \mathrm{~mm}$ is thermal emission from a dust core around the source.

The temperature of the dust core can be derived from the SED. For simplicity, we assume a constant temperature for the dust core, and the solid angle subtended by the dust core, $\Omega$, is independent of frequency, $\nu$, so that the observed flux is given by

$$
F_{\nu}=\Omega\left(1-e^{-\tau_{\nu}}\right) B_{\nu}\left(T_{\text {dust }}\right),
$$

where the optical depth

$$
\tau_{\nu}=\kappa_{\nu} \sigma
$$

where $\kappa_{\nu}$ is the mass opacity and $\sigma$ is the column density. With (see, e.g., Beckwith et al. 1990)

$$
\kappa_{\nu}=0.1\left(\frac{\nu}{10^{12} \mathrm{~Hz}}\right)^{\beta} \mathrm{cm}^{2} \mathrm{~g}^{-1},
$$

the optical depth can be simplified as

$$
\tau_{\nu}=\left(\frac{\nu}{\nu_{0}}\right)^{\beta}
$$

with an optical depth of unity at $\nu_{0}$. As can be seen from the SED, the emission is optically thin at $\lambda=1.1$ and $1.33 \mathrm{~mm}$, and their flux ratio implies that $\beta \sim 1$. The solid angle $\Omega$ is uncertain, likely $<2$ ". $3 \times 1^{\prime \prime}$ (see Fig. 1a). With $\Omega=1.2$ and $2.3 \operatorname{arcsec}^{2}$, the SED can be fitted with $T_{\text {dust }}=47.6 \mathrm{~K}$ and $\nu_{0}=3 \times 10^{12} \mathrm{~Hz}$ and $T_{\text {dust }}=45.4 \mathrm{~K}$ and $\nu_{0}=6 \times 10^{12} \mathrm{~Hz}$, respectively (see Fig. $1 b$ ). Thus, $T_{\text {dust }}$ is assumed to be $46 \mathrm{~K}$. With this temperature and the optically thin emission at $\lambda=1.33 \mathrm{~mm}$, the (gas + dust) mass is estimated to be

$$
M \sim \frac{D^{2} F_{\nu}}{B_{\nu}\left(T_{\text {dust }}\right) \kappa_{\nu}} \sim 0.08 M_{\odot},
$$

where the distance to the source $D=460 \mathrm{pc}$.

$$
\text { 3.2. } \mathrm{C}^{18} \mathrm{O}(J=2-1) \text { Emission }
$$

In $\mathrm{C}^{18} \mathrm{O}$, an envelope is seen around the source in the equator between the diffuse nebulae (Fig. $2 a$ ). Faint shells ( $2 \sigma$ contours) are also seen opening to the north and south surrounding the bases of the nebulae. The envelope is better seen in the blueshifted emission, in which the shells are much fainter than the envelope (Fig. $2 b$ ). It is extended across the source from the east to the west with a position angle of $\sim 110^{\circ} \pm 10^{\circ}$, perpendicular to the jet axis. It is flattened with an (deconvolved) FWHM extent of $\sim 4$."3 (or $2000 \mathrm{AU}$ at $460 \mathrm{pc}$ ) and an aspect ratio of $\sim 3: 2$. In the redshifted emission, the envelope is less extended. Since the redshifted emission around the bases of the nebulae is as bright as that of the envelope, the redshifted emission appears extended along the jet axis.

A cut along the major axis of the flattened envelope shows a position-velocity (PV) structure with the emission seen across the source with the redshifted and blueshifted peaks on either side of the source (Fig. 3). The blueshifted emission spreads across the source with a peak at $\left(-0.5 \mathrm{~km} \mathrm{~s}^{-1},-0.45\right)$ in the west, while the redshifted emission spreads across the source with a peak at $\left(0.35 \mathrm{~km} \mathrm{~s}^{-1}, 0\right.$ " 1$)$ in the east. This PV structure is similar to that seen in $\mathrm{C}^{18} \mathrm{O} J=1-0$ toward an edge-on dynamically infalling envelope with rotation around the young Class 0 source IRAS $04368+2557$ in L1527 (Ohashi et al. 1997). It suggests that the material of the flattened envelope is also infalling toward the source with rotation, with the redshifted emission from the near side and the blueshifted emission from the far side. 


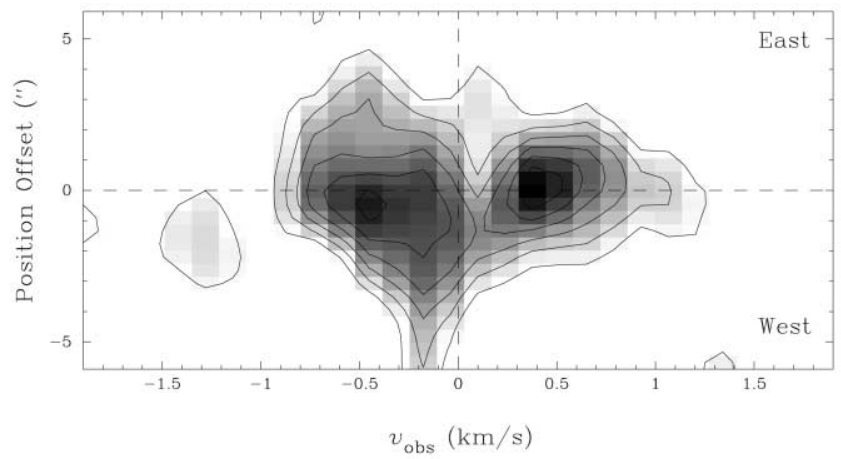

FIG. 3.- $\mathrm{PV}$ diagram of $\mathrm{C}^{18} \mathrm{O}$ emission cut along the major axis of the flattened envelope.

\section{3. ${ }^{13} \mathrm{CO}(J=2-1)$ Emission}

In ${ }^{13} \mathrm{CO}$, the emission peaks at the source and extends to the bases of the nebulae (Fig. $4 a$ ). The emission can be decomposed into low-velocity and high-velocity components (see Fig. $5 b$ and the explanation in the following paragraph). At low velocity, the emission forms an hourglass structure with a narrow waist around the source (Fig. $4 b$ ), with the blueshifted emission opening to the north and the redshifted emission opening to the south around the bases of the nebulae (Fig. 4c). Around the source, the blueshifted emission peaks a little to the west, while the redshifted emission shifts to the east of the source and is weak along the major axis of the flattened $\mathrm{C}^{18} \mathrm{O}$ envelope. At high velocity, the blueshifted emission peaks to the west while the redshifted emission peaks to the east of the source (Fig. $4 d$ ). The emission also extends to the north and south along the jet axis.

A cut across the ${ }^{13} \mathrm{CO}$ waist along the major axis of the flattened $\mathrm{C}^{18} \mathrm{O}$ envelope shows that the ${ }^{13} \mathrm{CO}$ emission has a larger velocity range than that of the $\mathrm{C}^{18} \mathrm{O}$ emission (Figs. $5 a$ and $5 b$ ). The velocity range is thus decomposed into low- and highvelocity components, with the range of the low-velocity component similar to that of the $\mathrm{C}^{18} \mathrm{O}$ emission. At low velocity, the blueshifted emission is seen across the source along the major axis of the flattened envelope with a peak at $\left(-0.3 \mathrm{~km} \mathrm{~s}^{-1}, 0\right.$.' 1$)$, and a redshifted dip is seen around $0.4 \mathrm{~km} \mathrm{~s}^{-1}$ across the source along the major axis of the flattened envelope. This dip may be due to the absorption of a cold material infalling toward the source (Evans 1999), which is probably traced by the bright $\mathrm{C}^{18} \mathrm{O}$ emission around that velocity (see Fig. $5 b$ ). At high velocity, the

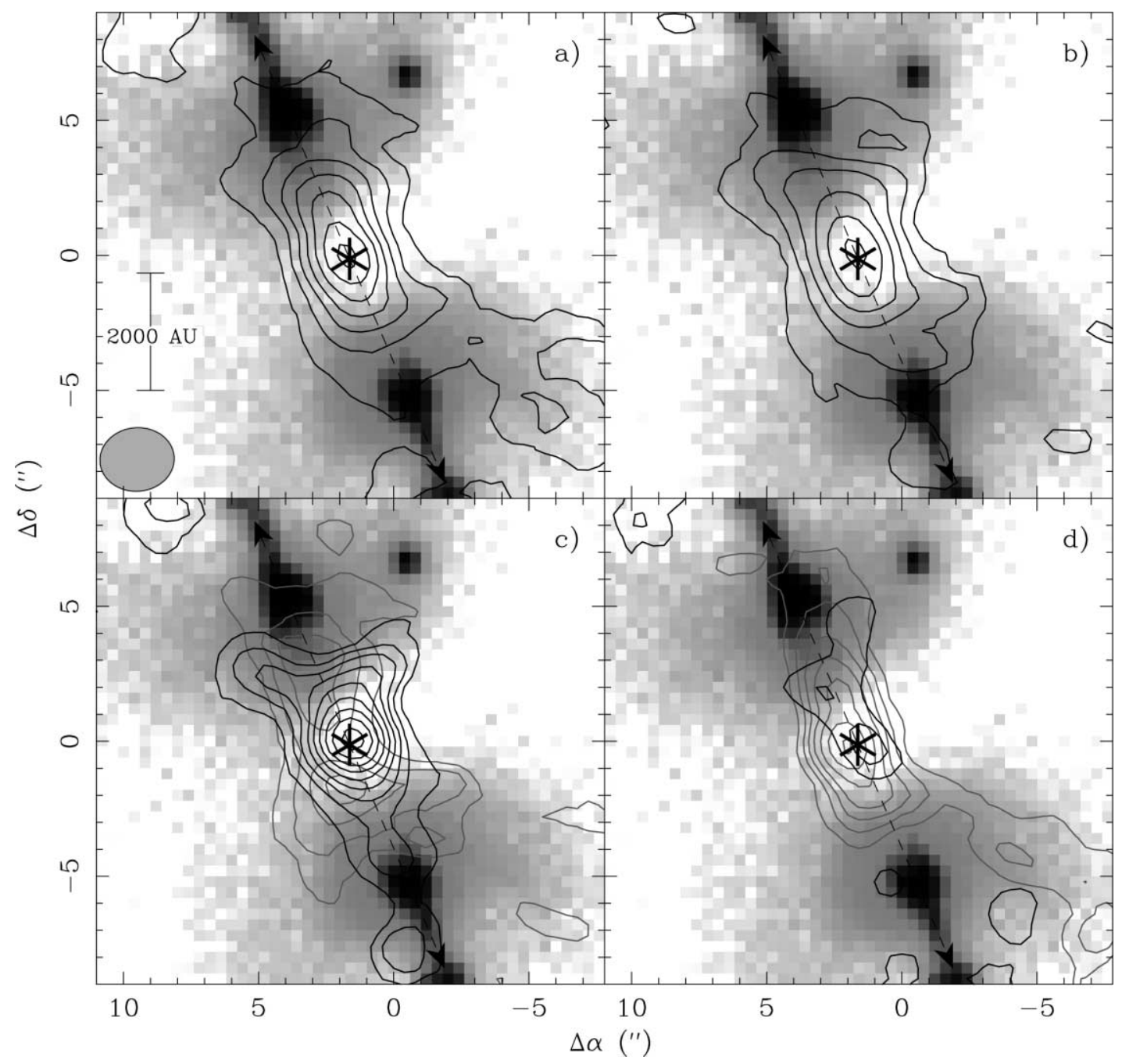

FIG. 4. $-{ }^{13} \mathrm{CO}$ emission plotted on top of the $\mathrm{H}_{2}$ image, with the asterisk indicating the IRAS source. (a) Total emission integrated over $3.4 \mathrm{~km} \mathrm{~s}{ }^{-1}$ from -1.7 to $1.7 \mathrm{~km} \mathrm{~s}^{-1}$. The contours go from 2 to $20 \sigma$ with a step of $3 \sigma$, where $\sigma=0.15 \mathrm{Jy}$ beam ${ }^{-1} \mathrm{~km} \mathrm{~s}^{-1}$. (b) Low-velocity emission integrated from -0.7 to $0.7 \mathrm{~km} \mathrm{~s}$ s $^{-1}$. The contours go from 2 to $17 \sigma$ with a step of $3 \sigma$, where $\sigma=0.09 \mathrm{Jy} \mathrm{beam}^{-1} \mathrm{~km} \mathrm{~s}^{-1}$. (c) Low-blueshifted (black contours) and low-redshifted (gray contours) emission, integrated from -0.7 to 0 and 0 to $0.7 \mathrm{~km} \mathrm{~s}^{-1}$, respectively. The contours go from 2 to $18 \sigma$ with a step of $2 \sigma$, where $\sigma=0.06 \mathrm{Jy} \mathrm{beam}{ }^{-1} \mathrm{~km} \mathrm{~s}^{-1}$. (d) Highblueshifted (black contours) and high-redshifted (gray contours) emission, integrated from -1.7 to -0.7 and 0.7 to $1.7 \mathrm{~km} \mathrm{~s}^{-1}$, respectively. The contours go from 2 to $14 \sigma$ with a step of $2 \sigma$, where $\sigma=0.08 \mathrm{Jy}^{\text {beam }}{ }^{-1} \mathrm{~km} \mathrm{~s}^{-1}$. [See the electronic edition of the Journal for a color version of this figure.] 


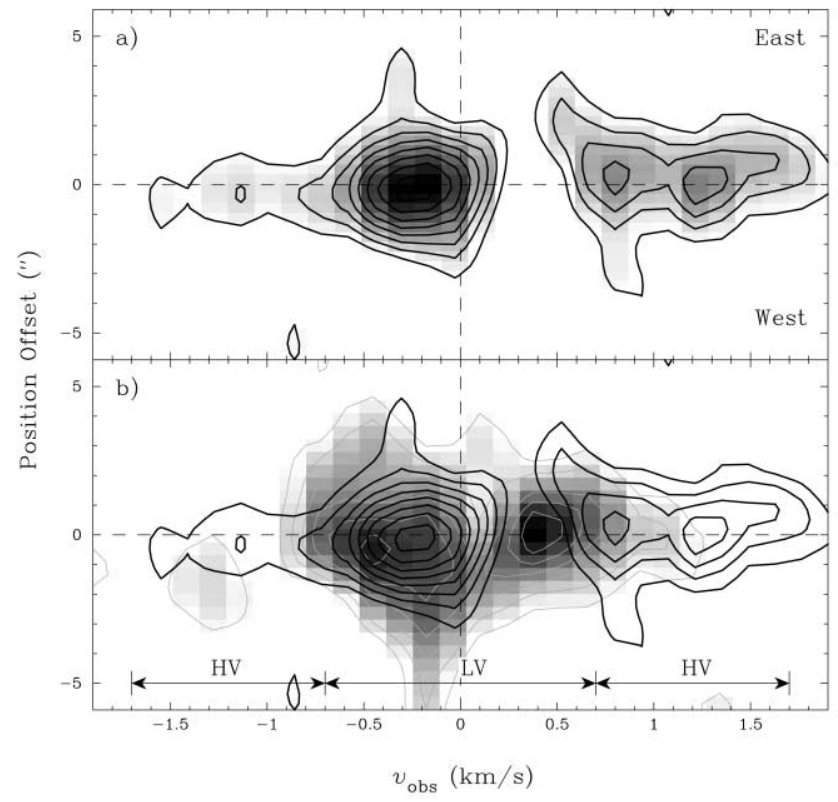

FIG. 5.- $\mathrm{PV}$ diagrams of $\mathrm{C}^{18} \mathrm{O}$ and ${ }^{13} \mathrm{CO}$ emission cut along the major axis of the flattened envelope. $(a){ }^{13} \mathrm{CO}$. (b) Overlay of the two. Here LV denotes low velocity and $\mathrm{HV}$ denotes high velocity.

redshifted and blueshifted emission are each confined on one side of the source, as seen in Figure 4d. This high-velocity emission is unlikely to be from outflow, with the redshifted and blueshifted emission on either side of the source. This high-velocity emission may trace the inner part of the envelope, where the rotation starts to dominate over the infall motion and a rotationally supported disk starts to form.

$$
\text { 3.4. }{ }^{12} \mathrm{CO}(J=2-1) \text { Emission }
$$

A ${ }^{12} \mathrm{CO}$ outflow is seen surrounding the $\mathrm{H}_{2}$ jet, with a narrow waist around the source (Fig. 6). Near the source, limb-brightened outflow shells are seen opening to the north and south from the source surrounding the $\mathrm{H}_{2}$ jet. The shells may extend farther down along the jet axis, connecting to the prominent $\mathrm{H}_{2}$ bow shocks SB4 and NB3, as seen in the lower $J$ transition line of ${ }^{12} \mathrm{CO}$ (Lee et al. 2000). Deeper observations with shorter baselines are needed to confirm this. At high velocities, the ${ }^{12} \mathrm{CO}$ emission is jetlike, i.e., collimated and knotty, much like the $\mathrm{H}_{2}$ jet, located inside the cavity defined by the diffuse nebulae and ${ }^{12} \mathrm{CO}$ outflow shells (Fig. $6 b$ ). In the south, the ${ }^{12} \mathrm{CO}$ outflow shell is seen coincident with the $\mathrm{H}_{2}$ bow shock SB4, probably tracing the ambient material swept up by the $\mathrm{H}_{2}$ bow shock. Note that here the ${ }^{12} \mathrm{CO}$ outflow shell is mainly seen on one side of the bow shock, which is brighter in $\mathrm{H}_{2}$.

Assuming an optically thin emission, a temperature of $50 \mathrm{~K}$, and a ${ }^{12} \mathrm{CO}$ abundance of $8.5 \times 10^{-5}$ with respect to molecular hydrogen (Frerking et al. 1982), the $\mathrm{H}_{2}$ column density of the shells around the source and the bow shock SB4 is found to be $\sim 10^{20} \mathrm{~cm}^{-2}$. Assuming that the shell thickness is $\sim 3^{\prime \prime}$ (see Fig. 6), the density is $\sim 5 \times 10^{3} \mathrm{~cm}^{-3}$. The peak column density along the jet axis is $\sim 5 \times 10^{20} \mathrm{~cm}^{-2}$. Assuming that the thickness is $\sim 2^{\prime \prime}$, the density is $\sim 4 \times 10^{4} \mathrm{~cm}^{-3}$. Note that due to the absorption of the ambient cloud, that part of the emission is optically thick, and that part of the emission is resolved out by the interferometer, the values presented here are lower limits of the true values.
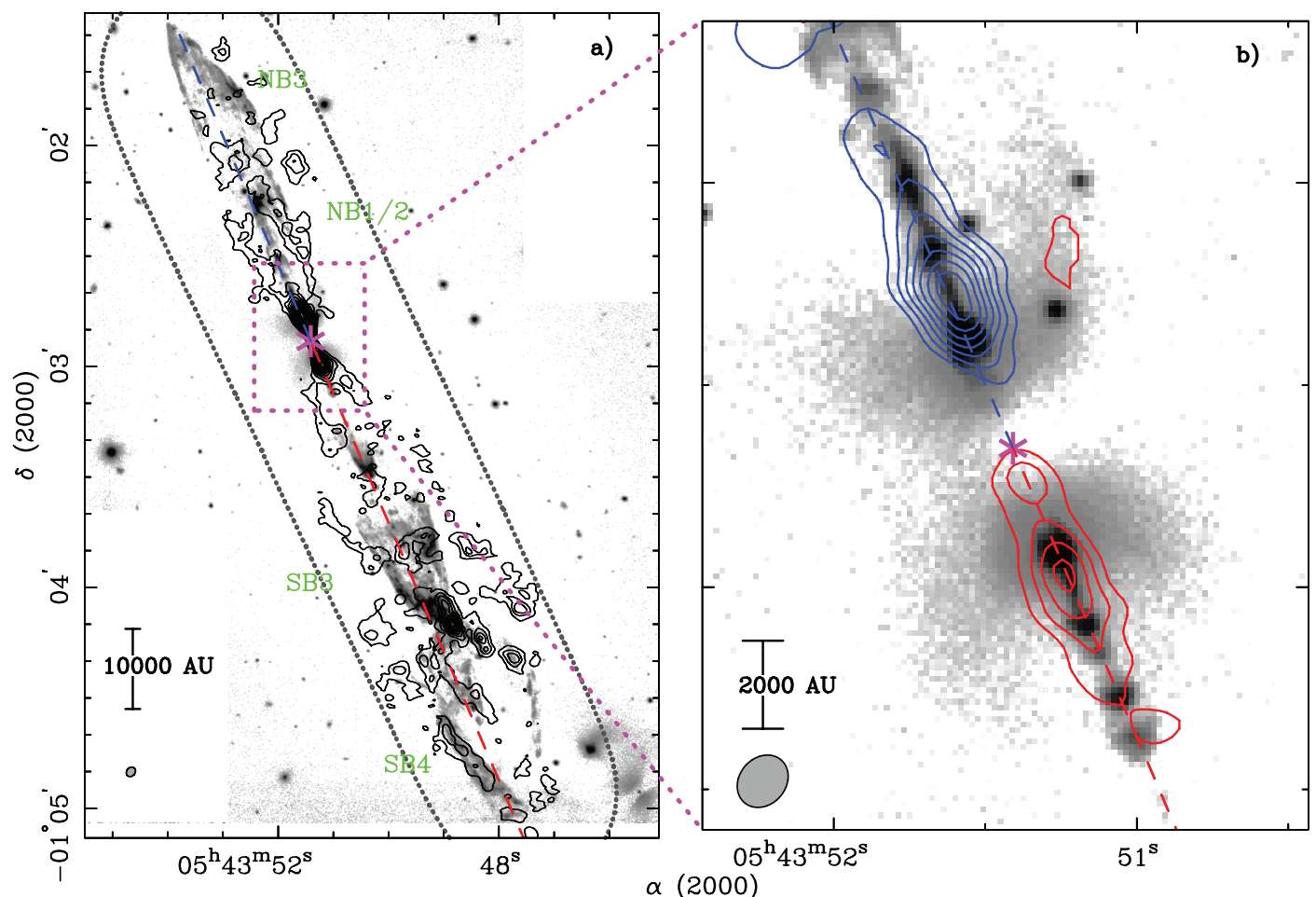

Fig. 6. - ${ }^{12} \mathrm{CO}$ emission plotted on top of the $\mathrm{H}_{2}$ image adopted from McCaughrean et al. (2002), with the asterisk indicating the IRAS source. The black dotted line outlines the observed region in our observations. (a) Black contours: Total emission integrated over $32 \mathrm{~km} \mathrm{~s}^{-1}$ from -16 to $16 \mathrm{~km} \mathrm{~s}{ }^{-1}$. The contours go from 2 to $24 \sigma$ with a step of $2 \sigma$, where $\sigma=0.68 \mathrm{Jy} \mathrm{beam}^{-1} \mathrm{~km} \mathrm{~s}^{-1}$. (b) Blue contours: High-blueshifted emission integrated from -5 to $-16 \mathrm{~km} \mathrm{~s}$. The contours go from 3 to $21 \sigma$ with a step of $3 \sigma$, where $\sigma=0.42 \mathrm{Jy} \mathrm{beam}^{-1} \mathrm{~km} \mathrm{~s}^{-1}$. Red contours: High-redshifted emission integrated from 2 to $13 \mathrm{~km} \mathrm{~s} \mathrm{~s}^{-1}$. The contours go from 3 to $12 \sigma$ with a step of $3 \sigma$, where $\sigma=0.42 \mathrm{Jy}_{\text {beam }}^{-1} \mathrm{~km} \mathrm{~s}^{-1}$. Here the velocity ranges are selected to show the jetlike structures as much as possible, without the confusion from the shell emission. 


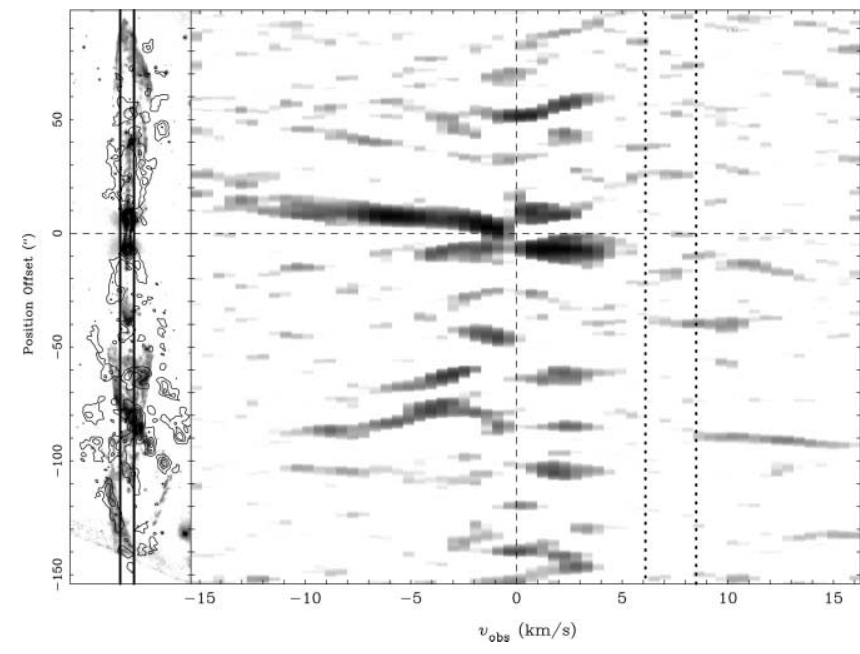

FIG. 7.-Left: $\mathrm{H}_{2}$ jet and $\mathrm{CO}$ outflow, with the outflow axis rotated to be aligned with the $y$-axis. The two vertical lines indicate the width of the cut for the PV diagram. Right: PV diagram of the CO outflow cut along the outflow axis. The two dotted lines around $7 \mathrm{~km} \mathrm{~s}^{-1}$ indicate the velocity range of a foreground ambient cloud seen by the Five College Radio Astronomy Observatory single-dish telescope (Lee et al. 2000).

This outflow, which is almost in the plane of the sky, is one of the best candidates for studying the outflow kinematics transverse to the jet axis. A cut along the jet axis shows a series of convex spur PV structures on both redshifted and blueshifted sides with the highest velocity near the $\mathrm{H}_{2}$ bow tips and knots (Fig. 7). Notice that the redshifted emission around $\sim 7 \mathrm{~km} \mathrm{~s}^{-1}$ merges with the foreground ambient cloud emission in velocity and thus is mostly resolved out from our observations. These PV structures are similar to those seen in the lower $J$ transition line of ${ }^{12} \mathrm{CO}$ (Lee et al. 2000), but with a higher velocity around the $\mathrm{H}_{2}$ knots and bow tips. These PV structures suggest that the outflow is accelerated by the shocks localized at the $\mathrm{H}_{2}$ bow tips and knots, so the velocity of the outflow decreases rapidly away from the tips, similar to that seen in a pulsed jet simulation (see, e.g., Lee et al. 2001).

$$
\text { 3.5. SO }\left(N_{J}=5_{6}-4_{5}\right) \text { Emission }
$$

$\mathrm{SO}$ emission is detected around the source and the $\mathrm{H}_{2}$ knots in the south, especially the knot SK4 (Fig. 8a). Around the source, the emission is not well resolved and is slightly elongated with a major axis about midway between the jet and envelope axes. The blueshifted emission is to the north, while the redshifted emission is to the south of the source (Fig. $8 b$ ), similar to that seen in the $\mathrm{H}_{2}$ jet, suggesting that the SO emission may arise from interactions with the jet. The peaks of the redshifted and blueshifted emission, however, are a little off the jet axis, with the redshifted one shifted to the east and the blueshifted one shifted to the west, suggesting that the SO emission may also arise from the envelope. Around the knot SK4, the redshifted emission is to the east while the blueshifted emission is to the west, similar to that seen in the flattened $\mathrm{C}^{18} \mathrm{O}$ envelope.

The SO emission around the source may consist of more than one component. A cut along the jet axis shows two peaks at high velocity, one blueshifted at $\left(-5.5 \mathrm{~km} \mathrm{~s}^{-1}, 00^{\prime \prime} 64\right)$ in the north and one redshifted at $\left(6.1 \mathrm{~km} \mathrm{~s}^{-1},-0.53\right)$ in the south (Fig. 9a). The rapid increase in the velocity suggests that the $\mathrm{SO}$ emission at high velocity indeed arises from shock interactions with the jet. However, the SO emission seen around the systemic velocity may not be produced by the shock interactions, because of its low velocity. A cut across the jet axis shows a rather symmetric PV structure about the source (Fig. 9b) at low velocity. The spatial extent and the velocity distribution (i.e., the redshifted emission is to the east and the blueshifted emission is to the west) of this PV structure are similar to that of the flattened $\mathrm{C}^{18} \mathrm{O}$ envelope, suggesting that the $\mathrm{SO}$ emission at low velocity may

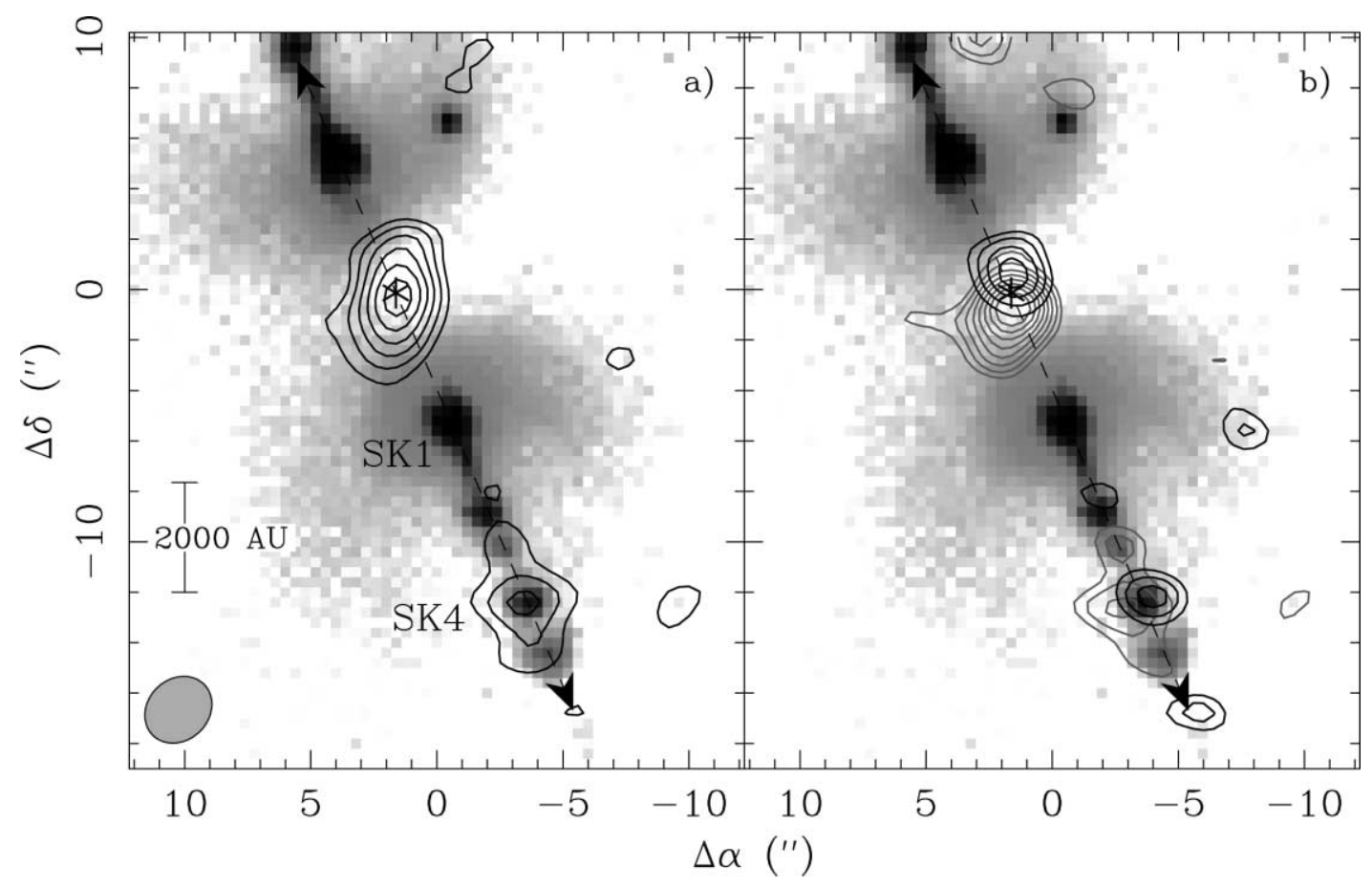

FIG. 8. - SO emission plotted on top of the $\mathrm{H}_{2}$ image, with the asterisk indicating the $I R A S$ source. $(a)$ Total emission integrated over $16 \mathrm{~km} \mathrm{~s}{ }^{-1}$ from -7 to $9 \mathrm{~km} \mathrm{~s}^{-1}$. The contours go from 3 to $13 \sigma$ with a step of $2 \sigma$, where $\sigma=0.35 \mathrm{Jy}_{\text {beam }}^{-1} \mathrm{~km} \mathrm{~s}^{-1}$. (b) Redshifted (gray contours) and blueshifted (black contours) emission,

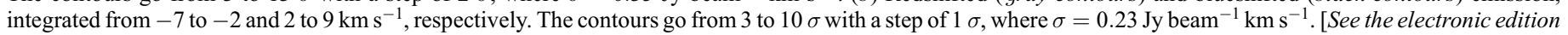
of the Journal for a color version of this figure.] 


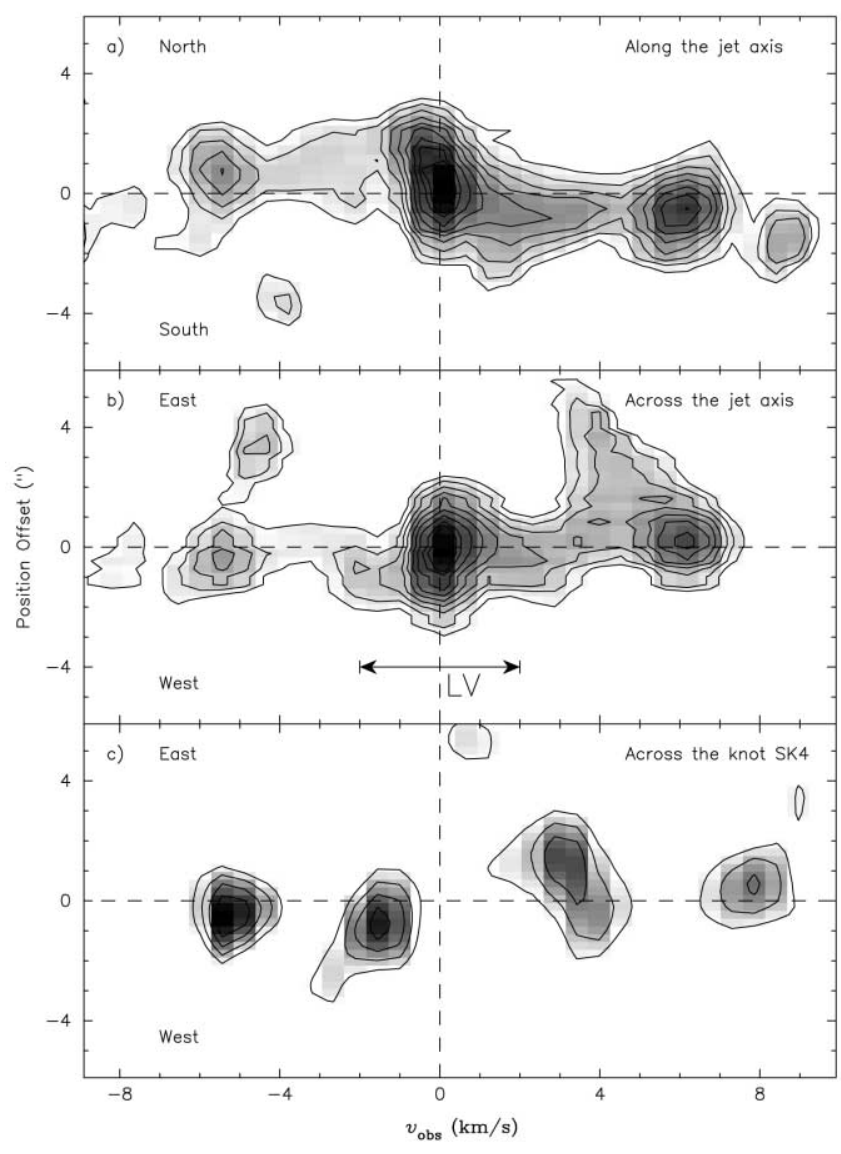

FIg. 9.-PV diagrams of SO emission. (a) Cut along the jet axis for the emission around the source. (b) Cut across the jet axis for the emission around the source. LV denotes low velocity. (c) Cut across the jet axis for the emission around the knot SK4. be associated with the flattened envelope. There is also highblueshifted and redshifted emission at $\sim 3^{\prime \prime}$ in the east.

A cut across SK4 shows that the redshifted emission is to the east, while the blueshifted emission is to the west (Fig. 9c), as seen in Figure $8 b$. The velocity extent seems to be similar to that around the source, with the higher velocity near the jet axis.

\subsection{Summary}

In this section, we summarize our observations with two composite figures, Figures $10 a$ and $10 b$. From these figures, it is clear that (1) the dust core seen in continuum and the ${ }^{13} \mathrm{CO}$ waist are surrounded by the flattened $\mathrm{C}^{18} \mathrm{O}$ envelope in the equator and thus may both trace the inner part of the envelope; (2) the ${ }^{13} \mathrm{CO}$ emission extends to the north and south from the source, surrounding the bases of the nebulae; (3) the ${ }^{12} \mathrm{CO}$ outflow appears as limbbrightened shells surrounding the $\mathrm{H}_{2}$ jet; and (4) the $\mathrm{SO}$ emission is detected around the source and the $\mathrm{H}_{2}$ knots in the south.

\section{MODELING OF CIRCUMSTELLAR ENVELOPE}

In the following, a simple edge-on disk model with both infall and rotation is used to reproduce the structures and kinematics of the flattened $\mathrm{C}^{18} \mathrm{O}$ envelope and the ${ }^{13} \mathrm{CO}$ waist seen around the source. In this model, the disk is assumed to have a constant thickness of $H$, an inner radius of $R_{\text {in }}$, and an outer radius of $R_{\text {out }}$. The number density of molecular hydrogen is assumed to be given by (in cylindrical coordinates)

$$
n=n_{0}\left(\frac{R}{R_{0}}\right)^{p},
$$

where $n_{0}$ is the density at a characteristic radius of $R_{0}=460 \mathrm{AU}$ (i.e., $1^{\prime \prime}$ ) and $p$ is a power-law index. The temperature of the envelope is uncertain and assumed to be given by

$$
T=T_{0}\left(\frac{R}{R_{0}}\right)^{q},
$$

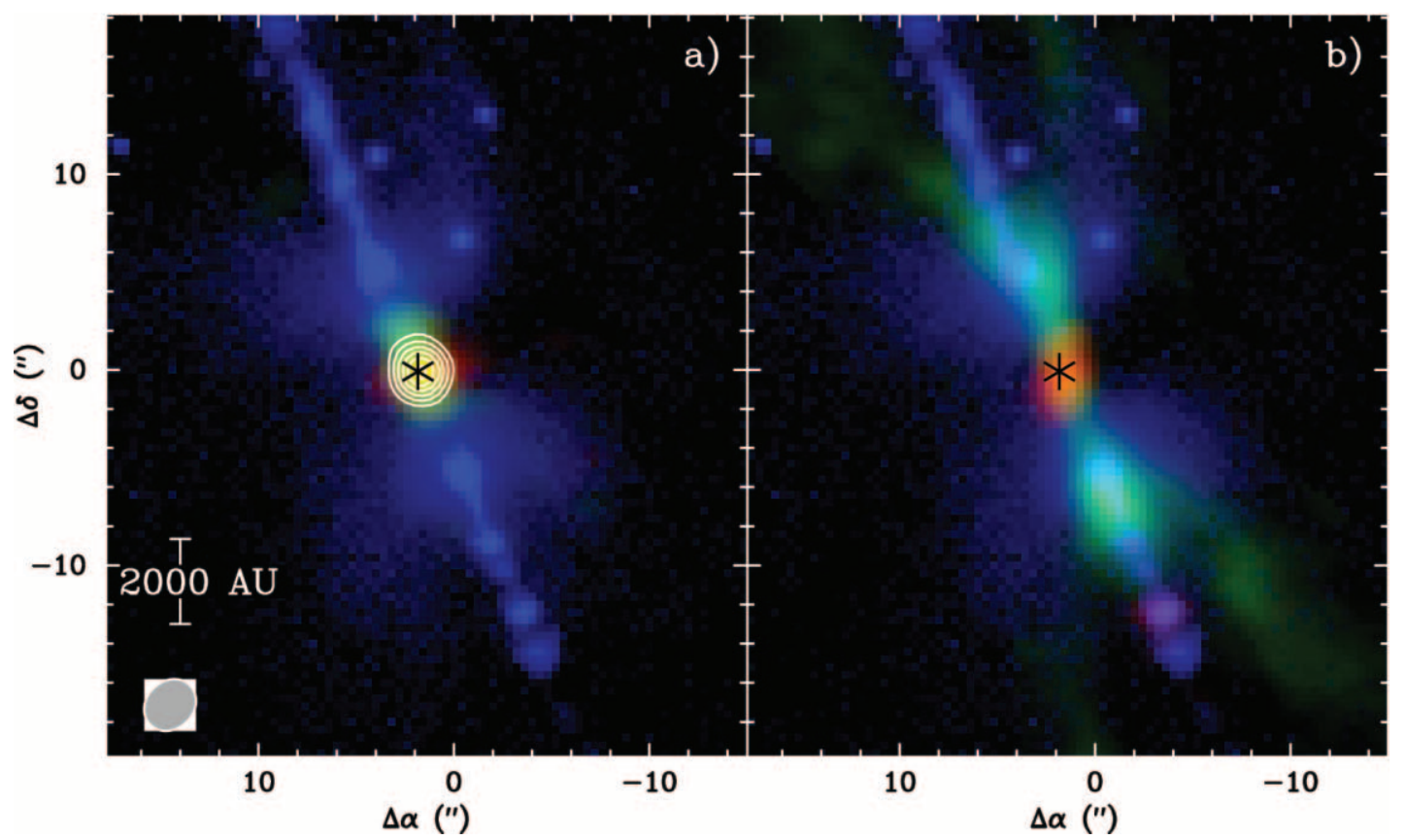

Fig. 10.-(a) Composite image of continuum (white contours), $\mathrm{C}^{18} \mathrm{O}($ red $),{ }^{13} \mathrm{CO}$ (green), and $\mathrm{H}_{2}$ (blue) emission. (b) Composite image of SO (red), ${ }^{12} \mathrm{CO}$ (green), and $\mathrm{H}_{2}$ (blue) emission. 
where $T_{0}$ is the temperature at $R_{0}$ and $q$ is a power-law index. The abundances of $\mathrm{C}^{18} \mathrm{O}$ and ${ }^{13} \mathrm{CO}$ relative to molecular hydrogen are assumed to be constant and given by $1.7 \times 10^{-7}$ and $9.5 \times 10^{-7}$, respectively (Frerking et al. 1982).

The detailed radial profiles of the infall velocity and the rotation velocity cannot be determined from our observations obtained at current spatial and velocity resolutions. For simplicity, we assume a dynamical infall that results in a free fall for the infall velocity. Assuming that the mass of the envelope is small compared with that of the source, we have

$$
v_{R}=\sqrt{\frac{2 G M_{*}}{R}} \sim 0.76 \sqrt{\frac{M_{*}}{0.15 M_{\odot}} \frac{R_{0}}{R}} \mathrm{~km} \mathrm{~s}^{-1},
$$

where $M_{*}$ is the mass of the source. The rotation seems differential with the velocity increasing toward the source (see Figs. 3 and 5). However, the rotation is unlikely to be Keplerian because the rotation velocity, as found in the following, is much smaller than the infall velocity. In a dynamically infalling envelope with slow rotation, the specific angular momentum of each gas element is considered to be conserved, until the infall motion shifts to the centrifugally supported motion around the radius where the rotation velocity is comparable to the infall velocity (see, e.g., Nakamura 2000). Thus, the rotation velocity is assumed to be given by

$$
v_{\phi}=v_{\phi 0} \frac{R_{0}}{R},
$$

where $v_{\phi 0}$ is the rotation velocity at $R_{0}$ and it depends on the initial angular momentum at the outer radius. In the model calculations, radiative transfer is used to calculate the emission, with an assumption of local thermal equilibrium. For simplicity, the line width is assumed to be given by the thermal line width only. The line width due to turbulence is not included in our model. The channel maps of the emission derived from the model are convolved with the observed beams and velocity resolutions and then used to make the integrated maps and PV diagrams.

There are nine parameters in this model: $H, R_{\text {in }}, R_{\text {out }}, n_{0}, p, T_{0}$, $q, M_{*}$, and $v_{\phi 0}$. To narrow our search, only two promising values of $p$ are considered: (1) $p=-1.5$, as found in many theoretical infalling models (see, e.g., Nakamura 2000) and $\mathrm{HCO}^{+}$observations around low-mass envelopes (Hogerheijde 2001), and (2) $p=-2.0$, as found in 850 and $450 \mu \mathrm{m}$ continuum observations of low-mass envelopes (Shirley et al. 2000). We find that these values of $p$ can both result in a reasonable fit to our observations too (see Fig. 11). In addition, the values of other parameters do not depend much on these values of $p$. In our fits, we have $H \sim$ $550 \mathrm{AU}(\sim 1 . " 2), R_{\text {in }} \sim 115 \mathrm{AU}(\sim 0.25), R_{\text {out }} \sim 2300 \mathrm{AU}\left(\sim 5^{\prime \prime}\right)$, $M_{*} \sim 0.15 M_{\odot}, v_{\phi 0} \sim 0.3 \mathrm{~km} \mathrm{~s}^{-1}$, and $n_{0} \sim 4 \times 10^{6} \mathrm{~cm}^{-3}$. The values of $T_{0}$ and $q$, however, are required to be different between $\mathrm{C}^{18} \mathrm{O}$ and ${ }^{13} \mathrm{CO}$ in order to fit the observations. We have $T_{0} \sim 35 \mathrm{~K}$ and $q \sim-0.4$ for $\mathrm{C}^{18} \mathrm{O}$, and $T_{0} \sim 23 \mathrm{~K}$ and $q \sim-1$ for ${ }^{13} \mathrm{CO}$. With these values of parameters, our model can roughly reproduce (1) the observed structures of the flattened $\mathrm{C}^{18} \mathrm{O}$ envelope and the ${ }^{13} \mathrm{CO}$ waist seen around the source, (2) the observed PV structures with the emission seen across the source with the redshifted and blueshifted peaks on either side of the source, and (3) the observed redshifted dip in ${ }^{13} \mathrm{CO}$. Note that in the observations, the emission extending to the north and south is likely from the envelope material affected by the outflow shells (see $\S 5.1$ ) and thus is not included in our model.
The mass of the flattened envelope, which is given by

$$
M_{e}=2 m_{\mathrm{H}} n_{0} H \int_{R_{\mathrm{in}}}^{R_{\mathrm{out}}}\left(\frac{R}{R_{0}}\right)^{p} 2 \pi R d R,
$$

is $\sim 0.028$ and $0.025 M_{\odot}$ for $p=-1.5$ and -2 , respectively. This mass, however, is a factor of 3 lower than that of the dust core. Note that the mass derived for the dust core could have an uncertainty with a factor of 5 due to the large uncertainty in the mass opacity (Beckwith et al. 1990). The infall rate, which is given by

$$
\begin{array}{r}
\dot{M}(R)=2 \pi R H n\left|v_{R}\right| 2 m_{\mathrm{H}}, \\
\text { is } \sim 6 \times 10^{-6} M_{\odot} \mathrm{yr}^{-1} \text { at } R_{0}=460 \mathrm{AU} .
\end{array}
$$

\section{DISCUSSION}

HH 212 is a highly collimated jet powered by a young Class 0 source, IRAS 05413-0104. We have mapped the jet and its source in $1.33 \mathrm{~mm}$ continuum, ${ }^{12} \mathrm{CO},{ }^{13} \mathrm{CO}, \mathrm{C}^{18} \mathrm{O}$, and $\mathrm{SO}$. In the following, we discuss our results in detail.

\subsection{Circumstellar Envelope}

A flattened envelope is seen in $\mathrm{C}^{18} \mathrm{O}$ around the source in the equator perpendicular to the jet axis, with its inner part seen in ${ }^{13} \mathrm{CO}$. The structure and kinematics of the envelope can be roughly reproduced by a simple edge-on disk model with both infall and rotation. In this model, the density of the disk is assumed to have a power-law index of $p=-1.5$ or -2 , as found in other low-mass envelopes (Shirley et al. 2000; Hogerheijde 2001). The temperature of $\mathrm{C}^{18} \mathrm{O}$ has a power-law index of $q \sim-0.4$, as found in other low-mass envelopes (Shirley et al. 2000; Hogerheijde 2001). However, in order to reproduce the compact waist and the redshifted dip in ${ }^{13} \mathrm{CO}$, the temperature of ${ }^{13} \mathrm{CO}$ is required to decrease with $q \sim-1$, which is faster than that of $\mathrm{C}^{18} \mathrm{O}$. Notice that in our model, the redshifted dip is assumed to be produced by the absorption of a cold material at the outer part of the envelope. However, the dip may be partly due to missing flux in our interferometric observations (Gueth et al. 1997). Further observations and modeling (e.g., with flared disk model, turbulence, and missing flux consideration) are needed to improve this.

The envelope seems dynamically infalling toward the source with slow rotation because the kinematics is found to be roughly consistent with a free fall toward the source plus a rotation of a constant specific angular momentum. If this is the case, the source has a mass of $M_{*} \sim 0.15 M_{\odot}$ and the infall rate is $\sim 6 \times$ $10^{-6} M_{\odot} \mathrm{yr}^{-1}$ at $R_{0}=460 \mathrm{AU}$, similar to those found in other flattened infalling envelopes with slow rotation around lowmass protostars (Ohashi et al. 1997; Momose et al. 1998). Assuming that the accretion rate is constant in the past and given by the infall rate, the accretion time will be $M_{*} / \dot{M} \sim 3 \times 10^{4} \mathrm{yr}$, as expected for a Class 0 source. Notice that in theoretical models (e.g., Shu et al. 2000), this accretion rate corresponds to an isothermal sound speed of $0.3 \mathrm{~km} \mathrm{~s}^{-1}$, or equivalently a temperature of $25 \mathrm{~K}$, which is consistent with our simple model. This dynamically infalling envelope with rotation is expected to form a rotationally supported disk at $R \sim 74 \mathrm{AU}\left(\sim 00^{\prime \prime} 16\right)$, at which the rotation velocity is comparable to the infall velocity (Hayashi et al. 1993; Lin et al. 1994). The ${ }^{13} \mathrm{CO}$ emission seen at high velocity may arise from this rotationally supported disk, with the redshifted emission and the blueshifted emission on either side of the source (Fig. $4 d$ ). 

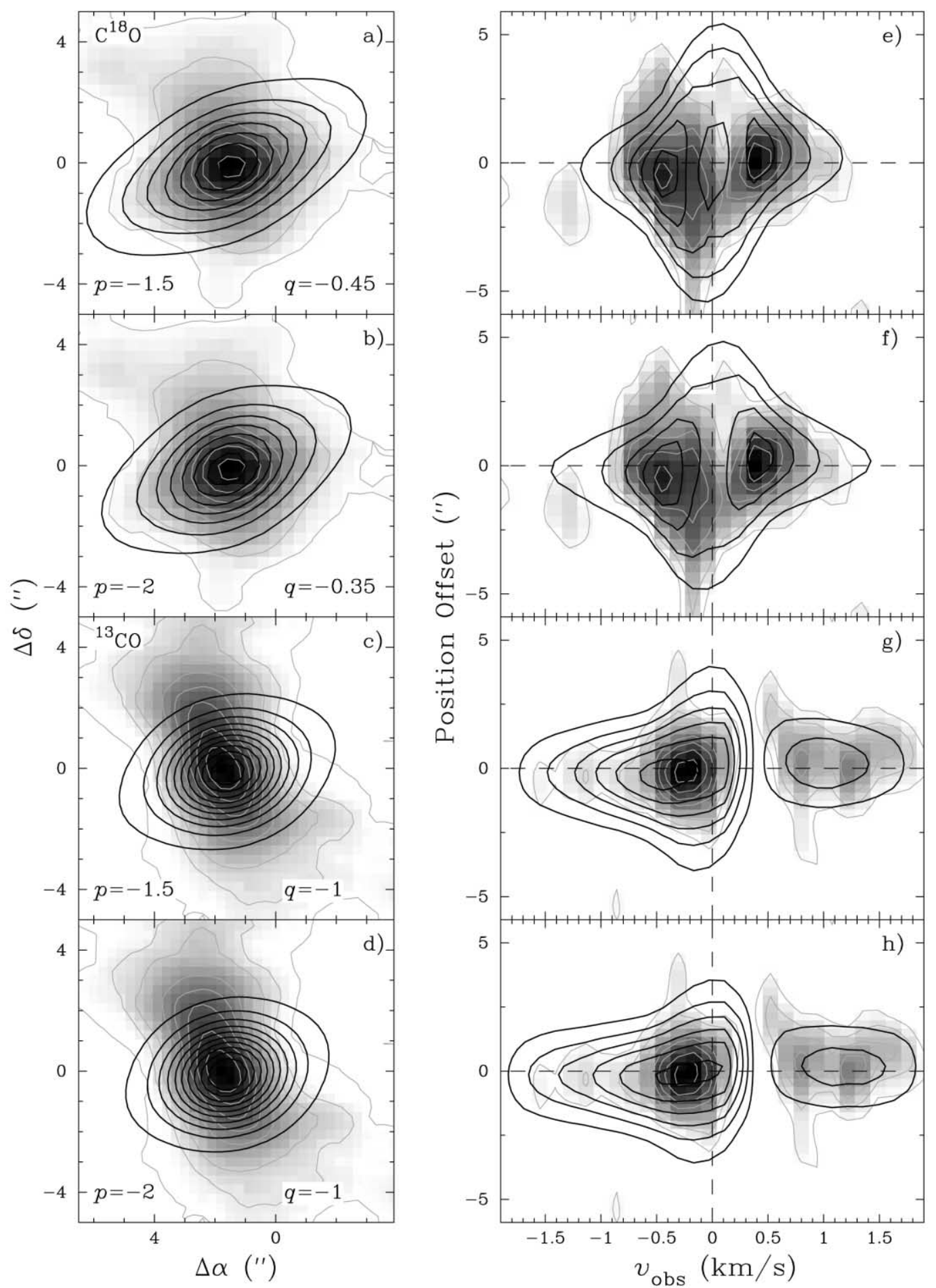

Fig. 11.-Modeling of envelope in $\mathrm{C}^{18} \mathrm{O}(a, b, e, f)$ and ${ }^{13} \mathrm{CO}(c, d, g, h)$. Gray contours with image are the observations; black contours are derived from our simple model. $(a-d)$, Total emission; $(e-h)$, PV diagrams cut along the major axis of the flattened envelope.

A flattened $\mathrm{NH}_{3}$ envelope has been seen rotating around the source in the same direction (Wiseman et al. 2001). It has a characteristic radius of $\sim 3500 \mathrm{AU}\left(\sim 8^{\prime \prime}\right)$ and can be considered to be the extension of the flattened $\mathrm{C}^{18} \mathrm{O}$ envelope into the surrounding material. At that radius, the infall velocity and rotation velocity are expected to be $\sim 0.27$ and $0.04 \mathrm{~km} \mathrm{~s}^{-1}$, respectively, in our simple model. This rotation velocity is consistent with that observed in the $\mathrm{NH}_{3}$ envelope, suggesting that the rotation of the $\mathrm{C}^{18} \mathrm{O}$ envelope is connected to that of the $\mathrm{NH}_{3}$ envelope and that the angular momentum is carried inward into the $\mathrm{C}^{18} \mathrm{O}$ envelope from the $\mathrm{NH}_{3}$ envelope. However, no infall velocity has been seen in the $\mathrm{NH}_{3}$ envelope. In theoretical models (e.g., Shu 1977), the infall radius is given by the isothermal sound speed times the accretion time. Assuming a temperature of $25 \mathrm{~K}$, the isothermal sound speed is $\sim 0.3 \mathrm{~km} \mathrm{~s}^{-1}$, resulting in an infall radius of $\sim 2000 \mathrm{AU}$, similar to the outer radius of the $\mathrm{C}^{18} \mathrm{O}$ envelope. Thus, it is possible that no infall motion is seen in the $\mathrm{NH}_{3}$ envelope. 
The flattened envelope is not formed by rotation because it is not rotationally supported, with the rotation velocity smaller than the infall velocity. Could it result from an interaction with the $\mathrm{CO}$ outflow? The flattened $\mathrm{NH}_{3}$ envelope is seen carved by the $\mathrm{CO}$ outflow, forming a bowl structure around it (Wiseman et al. 2001; Lee et al. 2000). In our observations, the shells seen in $\mathrm{C}^{18} \mathrm{O}$ and ${ }^{13} \mathrm{CO}$ around the bases of the nebulae may trace the (swept up) envelope material pushed by the outflow shells toward the equator, suggesting that the envelope around the source is also carved by the outflow. Having said that, the flattened envelope may not form by the outflow interaction because the shells are seen separated from the envelope.

Formation mechanisms of such a flattened infalling envelope around a forming star have been proposed with and without a magnetic field. Galli \& Shu (1993) examined the gravitational contraction of magnetized spherical cloud cores. They showed that flattened infalling envelopes are formed as the magnetic field impedes the contraction perpendicular to the field lines, which are aligned with the rotation axis. Hartmann et al. (1996) showed that even in the absence of a magnetic field, initially sheetlike cloud cores can also collapse to form flattened envelopes. Recently, Nakamura (2000) also showed that flattened infalling envelopes are a natural outcome of the gravitational contraction of prolate cloud cores with slow rotation. In this scenario, the flattened envelopes are predicted to have $n \propto r^{-1.5}$, $v_{r} \propto r^{-0.5}$, and $v_{\phi} \propto r^{-1}$, the same as our model.

\subsection{Molecular Outflow}

A ${ }^{12} \mathrm{CO}$ outflow is seen surrounding the $\mathrm{H}_{2}$ jet, with a narrow waist around the source. The outflow seen here in ${ }^{12} \mathrm{CO} J=2-1$ is similar to that seen in ${ }^{12} \mathrm{CO} J=1-0$, but with higher $J$ transition line of ${ }^{12} \mathrm{CO}$ tracing higher velocity around the knots and bow tips. The morphological relationship between the $\mathrm{H}_{2}$ jet and the ${ }^{12} \mathrm{CO}$ outflow, and the kinematics of the ${ }^{12} \mathrm{CO}$ outflow along the jet axis, are both consistent with those seen in a jetdriven bow shock model (see, e.g., Lee et al. 2001 and references therein). In that model, a highly collimated jet is launched from around the source, propagating into the ambient medium. A temporal variation in the jet velocity produces a chain of $\mathrm{H}_{2}$ knots propagating down along the jet axis. Since these $\mathrm{H}_{2}$ knots are localized (ballistic) shocks with high thermal pressure, they expand sideways, growing into $\mathrm{H}_{2}$ bow shocks with the shock velocity (and thus temperature) decreasing rapidly away from the bow tips. As these bow shocks propagate down along the jet axis, they sweep up the ambient material into a thin outflow shell around the jet axis. In our observations, the ${ }^{12} \mathrm{CO}$ shells around the source can be identified as the ambient material swept up by the $\mathrm{H}_{2}$ bow shocks. In addition, ${ }^{12} \mathrm{CO}$ emission also arises from around the $\mathrm{H}_{2}$ knots and bow shocks, with the velocity decreasing rapidly away from the knots and bow tips. Higher $J$ transition line of ${ }^{12} \mathrm{CO}$ traces higher velocity around the knots and bow tips, likely because it arises from regions of higher temperature and thus higher velocity closer to the knots and bow tips. As a result, the ${ }^{12} \mathrm{CO}$ emission appears jetlike at high velocities in high $J$ transition. Located inside the outflow cavity, the jetlike ${ }^{12} \mathrm{CO}$ emission is unlikely from the entrained ambient gas. The jet is probably intrinsically molecular, such as that seen in HH 211 (Gueth \& Guilloteau 1999). Since the shell is the swept up ambient material, the density of the ambient material is expected to be lower than that of the shell. Thus, the jet is an overdense jet with the density higher than that of the ambient material.

In the south, ${ }^{12} \mathrm{CO}$ emission is seen coincident with the $\mathrm{H}_{2}$ bow shock SB4, indicating that the shocked material there must have cooled very fast from $\sim 2000 \mathrm{~K}\left(\mathrm{H}_{2}\right)$ to a few $\times 10 \mathrm{~K}\left({ }^{12} \mathrm{CO}\right)$ because of a significant mixing with the ambient material. The transverse velocity there is also lower than that around other bow shocks (see Fig. 7), probably because the bow shock SB4 is sharing momentum with the quiescent ambient material (cf. Fig. 7 in Lee et al. 2001). Therefore, the bow shock SB4 may trace the leading bow shock where the head of the jet impacts the ambient material.

\subsection{SO Emission}

SO emission is seen around the source and the $\mathrm{H}_{2}$ knotty shocks in the south, especially the knot SK4. The emission around the source may arise from two components. Like that around the knot SK4, the emission seen at high velocity is likely to be from knotty shocks, which are expected to be there following the $\mathrm{H}_{2}$ knotty shocks toward the source. The shocks are not seen in $\mathrm{H}_{2}$, probably due to dust extinction. However, the SO emission seen around the systemic velocity may arise from the flattened envelope due to its low velocity. Observations at high angular resolution are needed to confirm this.

SO emission has also been seen toward the Cep A-East outflows, tracing ambient material at $60-100 \mathrm{~K}$ and shocked gas at 70-180 K (Codella et al. 2005). Assuming an optically thin emission, a temperature of $120 \mathrm{~K}$, and a SO abundance of $10^{-9}$ relative to molecular hydrogen (Codella et al. 2005), the mass is found to be $\sim 0.4$ and $0.1 M_{\odot}$, respectively, around the source and the knot SK4. However, these masses are too large to be from knotty shocks. The abundance of SO must have been highly enhanced. Note that ${ }^{12} \mathrm{CO}$ emission is weak around the knot SK4 (see Fig. 6b), probably suggesting that ${ }^{12} \mathrm{CO}$ there is destroyed by the shock interaction. The detection of the SO emission is likely because of the abundance enhancement of SO due to the shock-triggered release of various molecules (e.g., $\mathrm{H}_{2} \mathrm{~S} / \mathrm{OCS} / \mathrm{S}$ ) from dust mantles and the subsequent chemical reactions in the warm gas. Since the abundance is enhanced only for a very short period of time, the SO emission traces regions of recent shock activity (Codella et al. 2005).

Around the knot SK4, the redshifted emission is seen to the east while the blueshifted emission is seen to the west, similar to that seen in the flattened $\mathrm{C}^{18} \mathrm{O}$ envelope. A similar velocity structure has been seen around the knot SK1 (see Fig. 8 for its location) in $\mathrm{H}_{2}$ and considered as tentative evidence that the knot SK1 is rotating (Davis et al. 2000). It was observed with three slits from east to west with a separation of $\sim 0$ ". 45 . The peak velocities with respect to the systemic velocity were found to be $+4.6,+2.9$, and $+2.3 \mathrm{~km} \mathrm{~s}^{-1}$, measured from east to west. Thus, assuming that the central velocity at the knot SK 1 is $\sim 3 \mathrm{~km} \mathrm{~s}^{-1}$, the redshifted emission is seen to the east while the blueshifted is seen to the west. A similar velocity structure is seen here around the knot SK4 in SO but with higher velocity. Does this suggest that the knot SK4 is also rotating and that the jet carries away angular momentum from the infalling envelope? The velocity here, however, is much larger than $v_{\phi 0}$ (i.e., $0.3 \mathrm{~km} \mathrm{~s}^{-1}$ ) and thus may be too large to be from rotation. In addition, the shocked material there is expected to have sideways expansion. Further observations at higher angular resolution are needed to study it.

\section{CONCLUSION}

We have mapped the $1.33 \mathrm{~mm}$ continuum, ${ }^{12} \mathrm{CO}(J=2-1)$, ${ }^{13} \mathrm{CO}(J=2-1), \mathrm{C}^{18} \mathrm{O}(J=2-1)$, and $\mathrm{SO}\left(N_{J}=5_{6}-4_{5}\right)$ emission around the protostellar jet $\mathrm{HH} 212$ and its central source, IRAS 05413-0104. A dust core is seen in the continuum around the source, with a temperature of $\sim 46 \mathrm{~K}$ and a mass of 
$\sim 0.08 M_{\odot}$. A flattened envelope is seen in $\mathrm{C}^{18} \mathrm{O}$ around the source in the equator perpendicular to the jet axis, with its inner part seen in ${ }^{13} \mathrm{CO}$. The structure and kinematics of the envelope can be roughly reproduced by a simple edge-on disk model with both infall and rotation. The flattened envelope seems dynamically infalling toward the source with slow rotation because the kinematics is found to be roughly consistent with a free fall toward the source plus a rotation of a constant specific angular momentum. $\mathrm{A}^{12} \mathrm{CO}$ outflow is seen surrounding the $\mathrm{H}_{2}$ jet, with a narrow waist around the source. Jetlike structures are also seen in ${ }^{12} \mathrm{CO}$ near the source aligned with the $\mathrm{H}_{2}$ jet at high velocities. The morphological relationship between the $\mathrm{H}_{2}$ jet and the ${ }^{12} \mathrm{CO}$ outflow, and the kinematics of the ${ }^{12} \mathrm{CO}$ outflow along the jet axis, are both consistent with those seen in a jet-driven bow shock model. SO emission is seen around the source and the $\mathrm{H}_{2}$ knotty shocks in the south, tracing shocked emission around them.

We thank the SMA staff for their efforts in running and maintaining the array. H. B. acknowledges financial support by the Emmy-Noether-Program of the Deutsche Forschungsgemeinschaft (DFG, grant BE2578/1).
Andre, P., Ward-Thompson, D., \& Barsony, M. 2000, in Protostars and Planets IV, ed. V. Mannings, A. P. Boss, \& S. S. Russell (Tucson: Univ. Arizona Press), 59

Beckwith, S. V. W., Sargent, A. I., Chini, R. S., \& Güsten, R. 1990, AJ, 99, 924 Chapman, N. L., Mundy, L. G., Lee, C.-F., \& White, S. M. 2002, BAAS, 34, 1133

Chini, R., Reipurth, B., Sievers, A., Ward-Thompson, D., Haslam, C. G. T., Kreysa, E., \& Lemke, R. 1997, A\&A, 325, 542

Claussen, M. J., Marvel, K. B., Wootten, A., \& Wilking, B. A. 1998, ApJ, 507, L79

Codella, C., Bachiller, R., Benedettini, M., Caselli, P., Viti, S., \& Wakelam, V. 2005, MNRAS, 361, 244

Davis, C. J., Berndsen, A., Smith, M. D., Chrysostomou, A., \& Hobson, J. 2000, MNRAS, 314, 241

Evans, N. J., II. 1999, ARA\&A, 37, 311

Frerking, M. A., Langer, W. D., \& Wilson, R. W. 1982, ApJ, 262, 590

Galli, D., \& Shu, F. H. 1993, ApJ, 417, 243

Galván-Madrid, R., Avila, R., \& Rodríguez, L. F. 2004, Rev. Mex. AA, 40, 31

Gibb, A. G., Richer, J. S., Chandler, C. J., \& Davis, C. J. 2004, ApJ, 603, 198

Gueth, F., \& Guilloteau, S. 1999, A\&A, 343, 571

Gueth, F., Guilloteau, S., Dutrey, A., \& Bachiller, R. 1997, A\&A, 323, 943

Hartmann, L., Calvet, N., \& Boss, A. 1996, ApJ, 464, 387

Hayashi, M., Ohashi, N., \& Miyama, S. M. 1993, ApJ, 418, L71

Ho, P. T. P., Moran, J. M., \& Lo, K. Y. 2004, ApJ, 616, L1
Hogerheijde, M. R. 2001, ApJ, 553, 618

Lee, C.-F., Mundy, L. G., Reipurth, B., Ostriker, E. C., \& Stone, J. M. 2000, ApJ, 542, 925

Lee, C.-F., Stone, J. M., Ostriker, E. C., \& Mundy, L. G. 2001, ApJ, 557, 429

Lin, D. N. C., Hayashi, M., Bell, K. R., \& Ohashi, N. 1994, ApJ, 435, 821

McCaughrean, M., Zinnecker, H., Andersen, M., Meeus, G., \& Lodieu, N. 2002, Messenger, 109, 28

Momose, M., Ohashi, N., Kawabe, R., Nakano, T., \& Hayashi, M. 1998, ApJ, 504,314

Nakamura, F. 2000, ApJ, 543, 291

Ohashi, N., Hayashi, M., Ho, P. T. P., \& Momose, M. 1997, ApJ, 475, 211

Shirley, Y. L., Evans, N. J., II, Rawlings, J. M. C., \& Gregersen, E. M. 2000, ApJS, 131, 249

Shu, F. H. 1977, ApJ, 214, 488

Shu, F. H., Adams, F. C., \& Lizano, S. 1987, ARA\&A, 25, 23

Shu, F. H., Najita, J., Shang, H., \& Li, Z.-Y. 2000, in Protostars and Planets IV, ed. V. Mannings, A. P. Boss, \& S. S. Russell (Tucson: Univ. Arizona Press), 789

Wiseman, J., Wootten, A., Zinnecker, H., \& McCaughrean, M. 2001, ApJ, 550, L87

Zinnecker, H., Bastien, P., Arcoragi, J.-P., \& Yorke, H. W. 1992, A\&A, 265, 726

Zinnecker, H., McCaughrean, M. J., \& Rayner, J. T. 1998, Nature, 394, 862 